\title{
Positron emission tomography/computed tomography using 2-deoxy-2-fluoro-18-fluoro-D-glucose peri-tumoral halo uptake layer method outperforms magnetic resonance imaging and ultrasound methods in tumor size measurement of breast cancer
}

\author{
YINGZHE WU ${ }^{1}$, YUEZHONG LU ${ }^{1}$, CHENTAO XU ${ }^{1}$ and BIN LIN ${ }^{2}$ \\ Departments of ${ }^{1}$ Radiology and ${ }^{2}$ Pharmacy, Changxing People's Hospital, Huzhou, Zhejiang 313100, P.R. China
}

Received April 18, 2019; Accepted February 20, 2020

DOI: 10.3892/ol.2020.11492

\begin{abstract}
As a non-invasive method, positron emission tomography (PET)/computed tomography (CT) using 2-deox $\mathrm{y}$-2-fluoro-18-fluoro-D-glucose (18F-FDG) is applied as a useful modality in the diagnosis of breast cancer. By evaluating glucose metabolism, this method can also be used in staging, restaging and post-therapeutic response evaluation. To evaluate the reliability of the ${ }^{18} \mathrm{~F}$-FDG PET/CT-based peri-tumoral halo uptake layer (PHL) method for assessing tumor size, a total of 79 female patients with breast cancer who underwent ${ }^{18} \mathrm{~F}$-FDG PET/CT, breast ultrasound and magnetic resonance imaging (MRI) evaluations were included in the present study. Upon examination by two independent nuclear medicine radiologists, tumor sizes were estimated by ${ }^{18} \mathrm{~F}-\mathrm{FDG}$ PET/CT using margins defined as the inner line of the PHL. Pathological tumor sizes were evaluated on the direction of largest diameter indicated by previous imaging examination, which were also utilized as final standards. Statistical analysis of the results suggested that ${ }^{18} \mathrm{~F}-\mathrm{FDG}$ PET/CT had a more linear correlation with pathology compared with breast ultrasound $\left(\mathrm{r}^{2}=0.89\right.$ vs. 0.73$)$ and MRI $\left(\mathrm{r}^{2}=0.89\right.$ vs. 0.69$)$ in terms of tumor size estimation, including a significantly lower bias in size difference relative to pathology. ${ }^{18} \mathrm{~F}-\mathrm{FDG}$ PET/CT also exhibited improved performance compared with breast ultrasound and MRI in T stage assessment. These results indicated
\end{abstract}

Correspondence to: Professor Bin Lin, Department of Pharmacy, Changxing People's Hospital, 66 Taihu Zhong Road, Huzhou, Zhejiang 313100, P.R. China

E-mail: 13487035146@163.com

Abbreviations: $\quad{ }^{18} \mathrm{~F}-\mathrm{FDG} \quad \mathrm{PET} / \mathrm{CT}$, positron emission tomography/computed tomography using 2-deoxy-2-fluoro-18-fluor o-D-glucose; PHL, peri-tumoral halo uptake layer; MRI, magnetic resonance imaging; ICC, intraclass correlation coefficient

Key words: magnetic resonance imaging, ultrasound, tumor size, breast cancer, 18F-FDG PET/CT that the ${ }^{18} \mathrm{~F}$-FDG PET/CT-based PHL method was superior to breast ultrasound and MRI, and that it provides sufficient reliability and high accuracy for measuring tumor size in patients with breast cancer.

\section{Introduction}

Breast cancer is the most commonly diagnosed cancer in women, with 1,671,149 newly cases in 2012, accounting for $25.1 \%$ of all types of cancer, as well as its significant cancer-associated mortality in developed and developing countries $(1,2)$. Estimating the condition of patients, including the size and stage of the tumor, and establishing scientific therapy are key to obtaining satisfactory therapeutic effects (3). A careful and complete examination prior to surgery is important for selection of the suitable operation method and for surgical planning (4). One of the most important pieces of information required from preoperative examination is the size and area of extension of the tumor, which is important for surgical treatment and chemo-radiotherapy (5). Thus, assessing tumor size and estimating the tumor stage is imperative in clinical practice. Although a variety of imaging modalities have been used to diagnose breast cancer, mammography remains the traditional standard method for diagnosing breast cancer (6), with a reported sensitivity of $85 \%$, decreasing to $68 \%$ in women with dense breasts (7). However, this method does not estimate tumor size well, and it exhibits certain limitations regarding the qualitative diagnosis of breast tumors (8). It is best suited for breast examination and cancer screening (9). Additionally, the question of whether mammography should be recommended or not to women between the ages of 40 and 50 years depends upon the publication of relevant clinical trial data due to test sensitivity, disease prevalence, cost of screening and consequences of a false-positive result $(10,11)$.

Although magnetic resonance imaging (MRI) has been widely used in clinical practice for years, the appropriate use of MRI in elderly patients with breast cancer remains unclear. It has been reported that MRI has the greatest benefit in women presenting with an occult primary cancer and minimal additional benefit in elderly patients with breast 
cancer undergoing MRI for extent of disease evaluation or in post-treatment surveillance (12). MRI exhibits a high resolution and has been reported to accurately estimate tumor size $(13,14)$. However, MRI has also been reported to overestimate tumor size, particularly in patients with tumor (T)2 or T3 stage $(15,16)$. Although, MRI displays high sensitivity and specificity in breast cancer detection, the above limitation cannot be ignored. Sonography is more widely used in clinical practice, albeit as a method of cancer screening rather than tumor size estimation (17). Positron emission tomography (PET)/computed tomography (CT) using 2-deoxy-2 fluoro-18-fluoro-D-glucose (18F-FDG) is a useful modality in the diagnosis of patients with breast cancer (18). By evaluating glucose metabolism, it can also be used in staging, restaging and post-therapeutic response evaluation $(16,19)$. In a previous study, ${ }^{18} \mathrm{~F}-\mathrm{FDG}$ PET/CT was considered inappropriate for evaluation of tumor size and not recommended as a priority option in clinical practice due to its poor spatial resolution, which limits clear delineation of the tumor boundary (20). However, other studies have demonstrated the advantage of ${ }^{18} \mathrm{~F}-\mathrm{FDG}$ PET/CT in tumor size estimation (21). To the best of our knowledge, few studies have compared the sensitivity and accuracy of ${ }^{18} \mathrm{~F}$-FDG PET/CT with other traditional imaging modalities such as sonography and MRI. Besides, Jun et al (22) found a distinct layer between the tumor and background activity using a 10-step color scale with specific window level settings and named the distinct layer the peritumoral halo layer (PHL). Based on 18F-FDG, Jun et al, proposed a volume measurement method using PHL (22). However, although the newly developed PHL method appears promising for accurate estimation of tumor size, it has not yet been fully validated.

This study aimed to evaluate the concordance between the ${ }^{18} \mathrm{~F}$-FDG PET/CT based peri-tumoral halo uptake layer (PHL) method and the pathologic size of breast cancer. By comparing the reliability and correlation of ${ }^{18} \mathrm{~F}-\mathrm{FDG}$ PET/CT, sonography and MRI in pathological tumor size assessment, experimental evidences were provided for the selection of imaging modalities in breast cancer diagnosis. In order to examine the factors associated with discordance size, the accuracy of ${ }^{18} \mathrm{~F}-\mathrm{FDG}$ PET/CT, sonography and MRI was also compared in different tumor sizes (T1-T3), histologic subgroups and intrinsic subtypes.

\section{Materials and methods}

Patients. A total of 79 female patients with breast cancer selected from the database of Changxing People's Hospital (Huzhou, China) were included in this retrospective study. The staging of breast cancer was performed according to the 6th Edition of the American Joint Committee on Cancer Staging Manual (23). The inclusion criteria included: i) Patients who had undergone ${ }^{18} \mathrm{~F}$-FDG PET/CT, preoperative sonography and MRI examinations prior to initial treatment between May 2015 and June 2018; ii) patients who underwent the aforementioned mentioned imaging modalities within 10 days of diagnosis prior to surgery, and iii) patients with complete medical history and stored paraffin sample of the tumor obtained from surgery. The average time point when patients finished imaging using different modalities was 3 days before surgery. The exclusion criteria included: i) Patients with a history of neo-adjuvant treatment; ii) patients who had undergone preoperative mammotomy; iii) patients with non-avid tumor and clustered tumors, and iv) patients with incomplete data or insufficient results (such as MRI without contrast enhancement and pathology results without exact tumor size). The present study was supported by the Ethics Committee of Changxing People's Hospital (Huzhou, China; approval no. CPH 201505). All the patients signed informed consent forms.

Diagnostic imaging examination. A high-resolution 5-12 MHz linear array transducer, VOLUSON 530 and 730D (Kretztechnik AG) was used with a width of $50 \mathrm{~mm}$ to perform sonography imaging and the results were recorded according to the estimation of two independent examiners based on a widely accepted standard described in a previous study (24). The echo-poor center of the lesion and the echogenic halo were considered for the measurement of tumor size. Sonography was carried out by 2 independent experienced, board-certified breast radiologists.

A MAGNETOM Verio 3.0T (Siemens AG) was used to perform MRI. All patients were imaged in the prone position with both breasts placed into a dedicated 16-channel breast coil. The MRI protocols included the following: i) Bilateral axial turbo-spin-echo fat-suppressed T2-weighted image (Time of repetition (TR) / Time of echo (TE), 4,630/70 msec; field of view, $320 \mathrm{~mm}$; slice thickness, $3 \mathrm{~mm}$ and number of excitations, 1); ii) axial turbo-spin-echo T1-weighted image (TR/TE, 736/9.1 msec; field of view, $320 \mathrm{~mm}$; slice thickness, $3 \mathrm{~mm}$ and number of excitations, 1); iii) diffusion-weighted images (TR/TE, 5,800/82 msec; field of view, $360 \mathrm{~mm}$; slice thickness, $3 \mathrm{~mm}$ and b values 0,400 and $800 \mathrm{sec} / \mathrm{mm}^{2}$ ) and iv) measurement of the apparent diffusion-coefficient value.

Two PET/CT scanners [DSTe 8, (GE Medical Systems) and Gemini 64, (Philips Medical Systems)] were used to perform ${ }^{18} \mathrm{~F}$-FDG PET/CT examinations. All the patients fasted for $6 \mathrm{~h}$ before scanning and serum glucose levels were measured prior to $18 \mathrm{~F}-\mathrm{FDG}$ injection (DSTe $8,0.2 \mathrm{mCi} / \mathrm{kg}$ and Gemini 64, $0.1 \mathrm{mCi} / \mathrm{kg}$ ). A CT scan was performed in all the patients $1 \mathrm{~h}$ after 18F-FDG injection and images were collected. Each patient was scanned from the base of the skull to the mid-thigh level. Following low-dose CT scanning to correct for attenuation, PET acquisition began immediately in the same anatomical position (3-dimensional mode, 1.5-2.5 min per bed position). The acquired images were reconstructed using an iterative ordered subsets expectation maximization algorithm and then transferred to Advantage Workstation 4.5 (GE Healthcare). According to the methods described in a previous study, the PHL results of the tumors were determined (22). Sonography, MRI and ${ }^{18} \mathrm{~F}-\mathrm{FDG}$ PET/CT were performed by 3 independent groups. Each group was blinded to the results of the other groups. The quality control of machines was also monitored by radiologists according to widely accepted standards in China (25-27). Quality control consisted of daily, weekly, monthly and seasonally quality examination. Daily and weekly examinations included self-examination of hardware equipment and fast calibration to maintain the accuracy and uniformity of PET/CT. Monthly and seasonally examinations included normalized correction of PET scanner's 
A

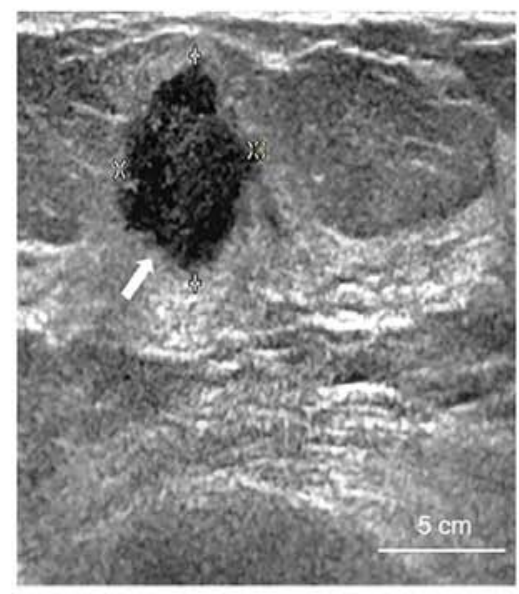

$\mathrm{B}$

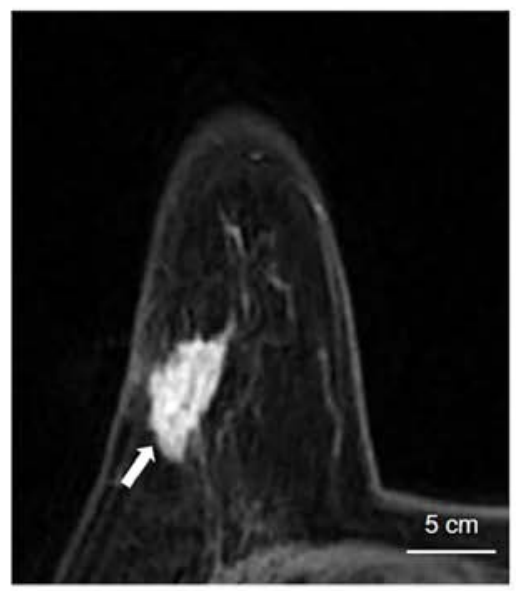

C

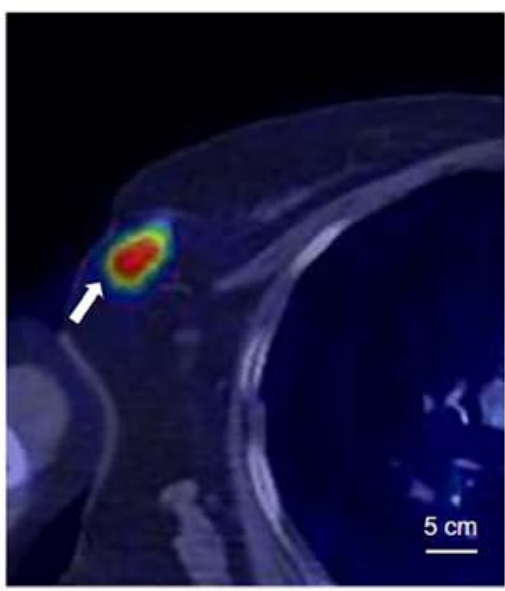

Figure 1. Imaging results of a 54-year-old patient with right breast cancer. (A) Breast sonography, (B) MRI (2 min dynamic image), (C) maximum intensity projection image of ${ }^{18} \mathrm{~F}$-FDG PET/CT. Arrows indicate the position of the tumor. MRI, magnetic resonance imaging; ${ }^{18} \mathrm{~F}-\mathrm{FDG}$ PET/CT, positron emission tomography/computed tomography using 2-deoxy-2-fluoro-18-fluoro-D-glucose.

sensitivity and image correction using a Well counter. The measurement of tumor size by sonography, MRI and ${ }^{18}$ F-FDG PET/CT was performed by two independent radiologists in each examination and the average tumor size from two independent radiologists were calculated as final result. Fig. 1 shows an example of sonography, MRI and ${ }^{18}$ F-FDG $\mathrm{PET} / \mathrm{CT}$ examination results of the representative case from a 54-year-old patient with right breast cancer.

Concordance between images and pathological results. Tumors were excised according to the direction of largest diameter indicated by previous imaging examination. The largest diameter from 2 edges of the paraffin sample were calculated as the tumor size. Pathological reports were completed by 2 independent pathologists from Department of Pathology, Changxing People's Hospital (Huzhou, China). The average diameter of tumors, recorded by two independent pathologists were defined as the standard of objective tumor size. The most widely accepted cut-off point of $0.5 \mathrm{~cm}$ described in previous studies was used in the current study to estimate the results of the imaging modalities $(9,28-30)$. The results were calculated using the image-derived tumor size minus the histopathologically determined tumor size and were considered to be concordant within $\pm 0.5 \mathrm{~cm}$. Values $<0.5 \mathrm{~cm}$ were graded underestimated, while those $>0.5 \mathrm{~cm}$ were graded overestimated regarding tumor size estimation.

Comparison of the results of the different imaging modalities among different subgroups of patients. The reliabilities of ${ }^{18} \mathrm{~F}-\mathrm{FDG} \mathrm{PET} / \mathrm{CT}$, sonography and MRI in terms of tumor size prediction were compared in different subgroups. Tumor size was classified into different subgroups as T1, T2 and T3. The imaging results for different histological subtypes were then compared with histopathologically derived tumor size.

Statistical analysis. All the collected data were described as mean \pm standard deviation. One-way ANOVA followed by Tukey's post-hoc test were performed for the evaluation of differences among 3 groups. Categorical variables were normally tested by the $\mathrm{r}^{2}$ test when appropriate. Kappa statistics were used to evaluate the inter-observer agreement for determination of PHL surrounding each tumor. Associations between pathology and MRI-determined tumor sizes, or between pathology and ${ }^{18} \mathrm{~F}$-FDG PET/CT-determined tumor sizes, were evaluated using linear regression analysis. Intraclass correlation coefficient (ICC) and Bland-Altman analyses were used to examine the concordance and reliability of tumor sizes obtained using MRI and ${ }^{18} \mathrm{~F}-\mathrm{FDG}$ PET/CT. Two-sided $\mathrm{P}<0.05$ were considered to indicate a statistically significant difference. Statistical analyses were conducted using SPSS v.19.0 software (IBM Corp.).

\section{Results}

Patient characteristics. A total of 79 Chinese female patients with breast cancer from Changxing People's Hospital were included in the current study. The clinicopathological characteristics and the results of tumor size estimation based on different imaging modalities are summarized in Table I. The age range of the patients was 42-76 years and the mean age was 53 years. Among all the patients, those with ductal adenocarcinoma were more numerous compared with non-ductal adenocarcinoma, and T2 was the most frequent tumor stage among patients. During ${ }^{18} \mathrm{~F}-\mathrm{FDG}$ PET/CT examination, the mean standardized uptake value (SUVmax) of the primary tumor was 4.80 (range, 1.12-19.61). The tumor sizes according to the results of sonography, MRI and ${ }^{18} \mathrm{~F}-\mathrm{FDG}$ PET/CT were $2.65,3.82$ and $2.98 \mathrm{~cm}$, respectively. The mean pathological size of the tumor was $3.05 \mathrm{~cm}$, ranging from $1.28-8.65 \mathrm{~cm}$, which was considered as the final standard size of each tumor.

PHL determination of tumors in patients with breast cancer. PHL, which was determined by 2 radiologist reviewers based on the results of ${ }^{18} \mathrm{~F}$-FDG PET/CT, exhibited good consistency with a contingency coefficient of $0.79(\mathrm{P}<0.01)$. The PHL of the majority of tumors detected was below the $40-50 \%$ band, while the area between 20 and $30 \%$ (olive-green color) of the band of SUVmax is the area where the PHL of each tumor was most commonly recorded (24\%; data not shown). The PHL exhibited a negative association with the SUVmax of 
Table I. Clinical characteristics of patients with breast cancer.

\begin{tabular}{|c|c|}
\hline Characteristics & No. of patients/mean (range) \\
\hline Number of patients & 79 \\
\hline Sex & Female \\
\hline Age, years & $53(42-76)$ \\
\hline \multicolumn{2}{|l|}{ Pathological type } \\
\hline Ductal & 68 \\
\hline Non-ductal & 11 \\
\hline Surgery & 48 \\
\hline Breast-conservation therapy & \\
\hline Mastectomy & 31 \\
\hline \multicolumn{2}{|l|}{ T stage } \\
\hline $\mathrm{T} 1$ & 23 \\
\hline $\mathrm{T} 2$ & 37 \\
\hline $\mathrm{T} 3$ & 19 \\
\hline \multicolumn{2}{|l|}{ Tumor size, $\mathrm{cm}$} \\
\hline Pathology & $3.05(1.28-8.65)$ \\
\hline Sonography & $2.65(0.95-7.58)$ \\
\hline MRI & $3.82(2.87-10.34)$ \\
\hline $\mathrm{PET} / \mathrm{CT}$ & $2.98(1.20-8.72)$ \\
\hline SUVmax of primary tumor & $4.80(1.12-19.61)$ \\
\hline
\end{tabular}

SUVmax, mean standardized uptake value; T, tumor stage.

separate tumors (Fig. 2), with the exception of 5 cases, as presented in Table II.

Concordance of tumor sizes measured different imaging modalities and pathology. All sonography, MRI and PET/CT examination results had statistically significant associations with pathological measurements regarding tumor size. Using the pathological size of the tumor as the final standard size, there were small differences observed between the imaging and pathology tumor size, namely sonography, $-0.78 \pm 1.10 \mathrm{~cm}$; MRI, $1.28 \pm 1.25 \mathrm{~cm}$ and ${ }^{18} \mathrm{~F}-\mathrm{FDG}$ PET/CT, $0.13 \pm 0.90 \mathrm{~cm}$, respectively (data not shown). The linear regression between ${ }^{18} \mathrm{~F}-\mathrm{FDG} \mathrm{PET} / \mathrm{CT}$ and pathology measurements $\left(\mathrm{r}^{2}=0.89 ; \mathrm{P}<0.01\right)$ was more significant compared with the linear correlation between sonography $\left(\mathrm{r}^{2}=0.73 ; \mathrm{P}<0.01\right)$ and MRI ( $\mathrm{r}^{2}=0.69 ; \mathrm{P}<0.01 ;$ Fig. 3).

In addition, ${ }^{18} \mathrm{~F}$-FDG PET/CT demonstrated a smaller bias in tumor size estimation compared with sonography and MRI in Bland-Altman analysis. ${ }^{18} \mathrm{~F}-\mathrm{FDG}$ PET/CT-derived tumor size exhibited a high concordance with pathological tumor size [0.95; 95\% confidence interval (CI), 0.92-0.97] using the ICC test, which was significantly superior to sonography $(0.83 ; 95 \%$ CI, 0.81-0.89) and MRI (0.77; 95\% CI, 0.65-0.84; Fig. 4).

Association of sonography, MRI and ${ }^{18}$ F-FDG PET/CT according to pathology $T$ stage in patients with breast cancer. In the analysis of subgroups classified by tumor $\mathrm{T}$ stage, ${ }^{18}$ F-FDG PET/CT compared with sonography or MRI demonstrated significantly lower size differences (vs. pathology) in

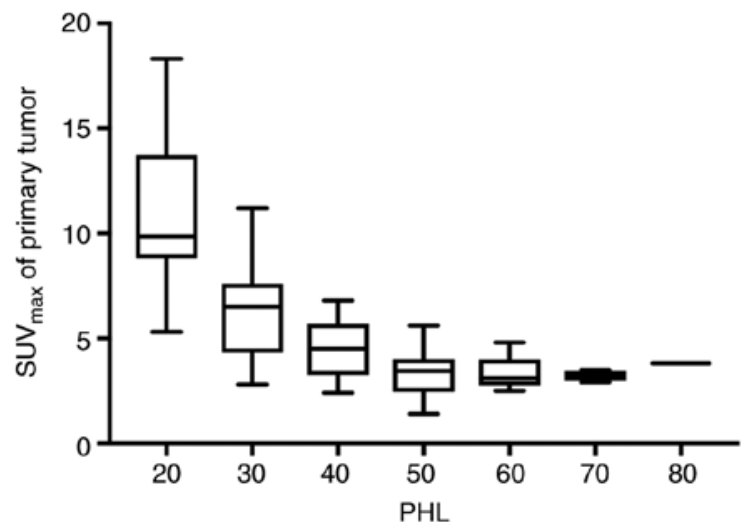

Figure 2. Association between SUVmax of primary tumor and PHL. PHL was calculated using Jun's method (21) by transforming PET/CT image into 10 -color scale, based on the SUVmax. PHL refers to the layer between tumor activity and background. PHL, peri-tumoral halo layer; SUVmax, mean standardized uptake value.

T2 and T3 stage tumors $(\mathrm{P}<0.05)$, but not in $\mathrm{T} 1$ stage tumors (Fig. 5). However, there were cases where $\mathrm{T}$ stage was wrongly estimated using imaging. Table III presents the $\mathrm{T}$ stages and tumor sizes of 12 patients whose $\mathrm{T}$ stages were incorrect on sonography, MRI or ${ }^{18} \mathrm{~F}-\mathrm{FDG}$ PET/CT. For sonography, there were 5 patients whose tumor $\mathrm{T}$ stages were overestimated ( 3 cases were considered T2 instead of T1 and 1 was considered T3 instead of T2; Table III). MRI-assessed T stages were incorrect for 7 patients, of which 5 cases were upstaged ( 3 cases from $\mathrm{T} 1$ to $\mathrm{T} 2 ; 1$ case from $\mathrm{T} 1$ to $\mathrm{T} 3$ and 1 case from $\mathrm{T} 2$ to $\mathrm{T} 3$; Table III) while 2 cases were down-staged ( 1 case from T3 to T2 and 1 case T2 to T1; Table III). A total of 2 patients were upstaged in ${ }^{18} \mathrm{~F}$-FDG PET/CT assessment (1 case was upstaged from T1 to T2 and 1 case from T2 to T3; Table III). Among the mismatched 12 cases, 1 case was incorrectly upstaged from T2 to T3 in sonography, MRI and ${ }^{18} \mathrm{~F}-\mathrm{FDG}$ PET/CT.

Additionally, ${ }^{18} \mathrm{~F}-\mathrm{FDG}$ PET/CT also demonstrated a significant advantage in accuracy of predicting tumor size compared with sonography and MRI in different pathological subgroups of tumors. The concordance rates for of sonography, MRI and ${ }^{18} \mathrm{~F}$-FDG PET/CT were 45.4, 31.8 and $69.2 \%$, respectively, in ductal adenocarcinoma, while for non-ductal adenocarcinoma, the concordance rates for sonography, MRI and ${ }^{18} \mathrm{~F}$-FDG PET/CT were 41.2, 29.6 and $72.5 \%$, respectively (data not shown).

\section{Discussion}

Previous studies have demonstrated that in early breast cancer, there is no association between long-term survival and mastectomy with tumors of relatively small size (5-9). In addition, complete removal of the tumor reduces the probability of recurrence (1). Thus, estimating the size and area of extension of the tumor accurately is important for chemoradiotherapy decision and surgical treatment. As a non-invasive method, ${ }^{18} \mathrm{~F}-\mathrm{FDG}$ PET/CT is applied in clinical practice. However, FDG PET/CT is not recommended as a priority option in clinical practice due to poor spatial resolution, which limits clear delineation of the tumor boundary. Studies comparing the reliability of ${ }^{18} \mathrm{~F}$-FDG PET/CT with MRI or sonography in terms 
Table II. Discordant cases $(\mathrm{n}=5)$ of PHL determined by 2 independent radiologists.

\begin{tabular}{|c|c|c|c|c|c|c|c|c|c|}
\hline \multirow[b]{2}{*}{ No. } & \multirow[b]{2}{*}{ Age } & \multirow[b]{2}{*}{ Histologic type } & \multirow{2}{*}{$\begin{array}{l}\text { SUVmax } \\
\text { of tumor }\end{array}$} & \multicolumn{2}{|c|}{ PHL (\%) } & \multicolumn{4}{|c|}{ Tumor size (mm) } \\
\hline & & & & Radiologist1 & Radiologist2 & Sonography & MRI & $\mathrm{PET} / \mathrm{CT}$ & Pathology \\
\hline 1 & 53 & Ductal adenocarcinoma & 8.2 & 30 & 20 & 7.8 & 8.0 & 7.5 & 7.2 \\
\hline 2 & 48 & Ductal adenocarcinoma & 3.2 & 70 & 60 & 2.4 & 3.6 & 2.7 & 2.9 \\
\hline 3 & 45 & Ductal adenocarcinoma & 3.8 & 50 & 60 & 3.1 & 2.9 & 3.8 & 3.5 \\
\hline 4 & 62 & Non-ductal adenocarcinoma & 2.1 & 70 & 80 & 2.2 & 2.8 & 2.3 & 1.8 \\
\hline 5 & 71 & Ductal adenocarcinoma & 4.6 & 60 & 70 & 4.2 & 4.3 & 5.1 & 4.9 \\
\hline
\end{tabular}

SUVmax, mean standardized uptake value; MRI, magnetic resonance imaging; PET/CT, positron emission tomography/computed tomography; MRI, magnetic resonance imaging; PHL, peri-tumoral halo uptake layer.
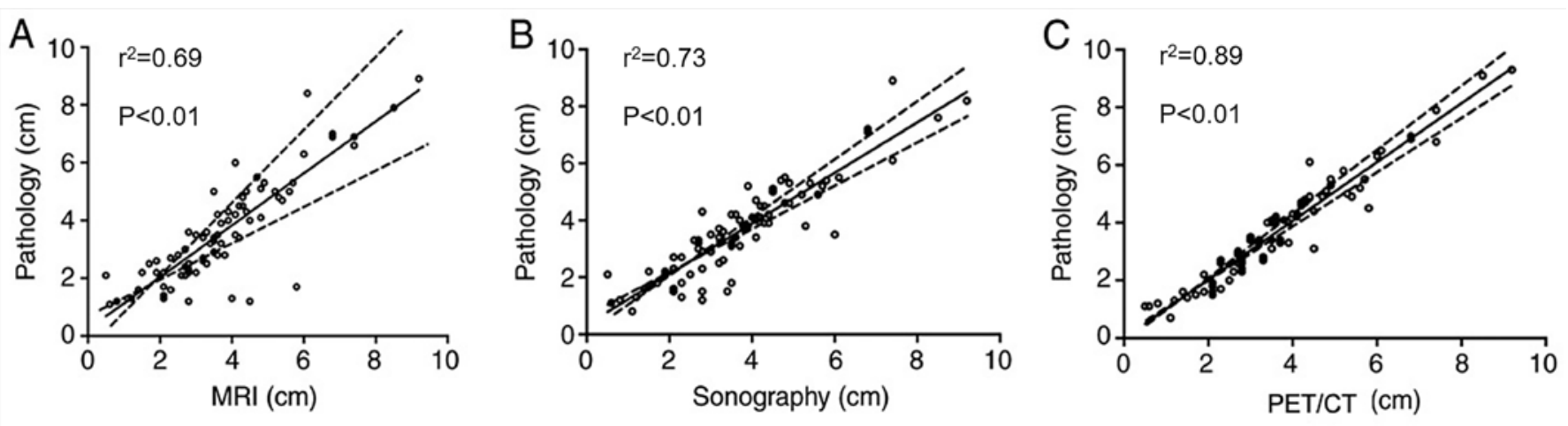

Figure 3. Linear regression analysis for tumor size in patients with breast cancer. (A) pathology vs. sonography, (B) pathology vs. MRI and (C) pathology vs. ${ }^{18} \mathrm{~F}-\mathrm{FDG} \mathrm{PET} / \mathrm{CT}$. MRI, magnetic resonance imaging; ${ }^{18} \mathrm{~F}-\mathrm{FDG} \mathrm{PET} / \mathrm{CT}$, positron emission tomography/computed tomography using 2-deoxy-2-fluoro-18-fluoro-D-glucose.
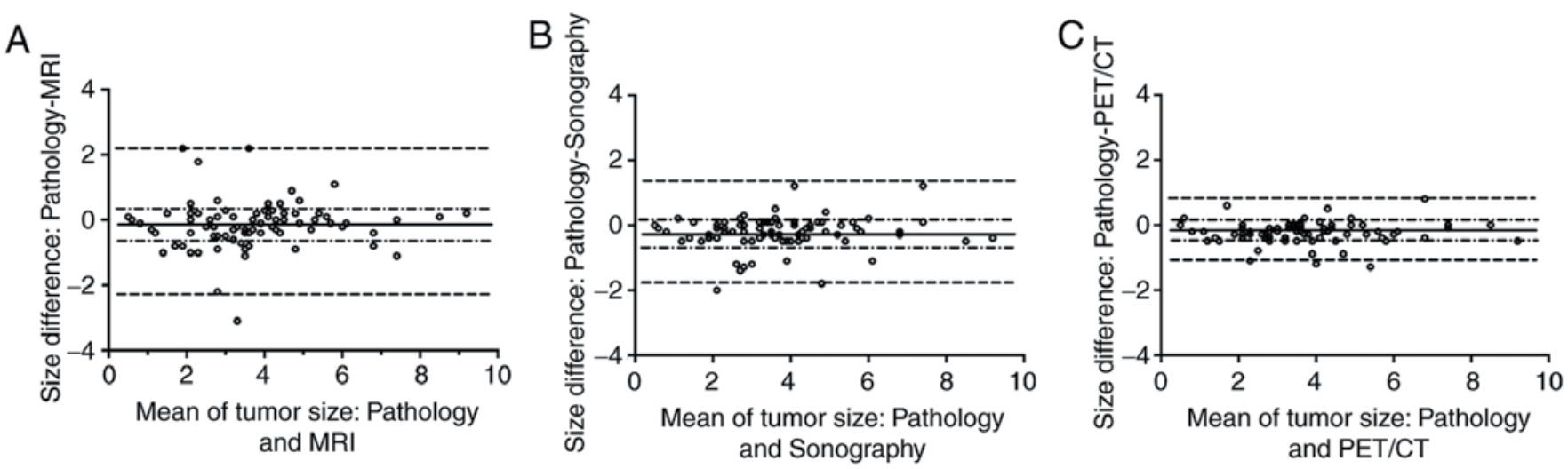

Figure 4. Bland-Altman plot analysis for tumor sizes. The $\mathrm{Y}$ axis, the difference between the two paired measurements; the $\mathrm{X}$ axis, the average of the two paired measures. (A) MRI vs pathology; (B) sonography vs pathology; (C) ${ }^{18} \mathrm{~F}-\mathrm{FDG}$ PET/CT vs pathology. MRI, magnetic resonance imaging; ${ }^{18} \mathrm{~F}-\mathrm{FDG}$ $\mathrm{PET} / \mathrm{CT}$, positron emission tomography/computed tomography using 2-deoxy-2-fluoro-18-fluoro-D-glucose.

of tumor size assessment of breast cancer are limited (20). The present study demonstrated that using PHL, the results from the ${ }^{18} \mathrm{~F}$-FDG PET/CT method had a higher accuracy and association with pathological tumor size compared with those obtained by MRI and sonography.

It has been previously reported that tumor size is often underestimated by sonography (31), which was also observed in the current study. Several hypotheses have been proposed to explain these discrepancies. Hieken et al (32), demonstrated that these discrepancies are derived from extensive intraductal in situ components caused by unclear margins of sonography. In the study by Gruber et al (31), a novel technique called panoramic mode, which allows a complete image to be built from individual sectional sonographic images, was used in order to obtain more accurate results of the tumor with the diameters exceeding the width of the transducer. This may 
Table III. Evaluation of 12 discordant cases of pathological T stage (22).

\begin{tabular}{|c|c|c|c|c|c|c|c|}
\hline \multirow[b]{2}{*}{ Serial no. } & \multicolumn{4}{|c|}{ T stage } & \multicolumn{3}{|c|}{ Size differences (vs. PET/CT, mm) } \\
\hline & Pathology & Sonography & MRI & $\mathrm{PET} / \mathrm{CT}$ & Sonography & MRI & $\mathrm{PET} / \mathrm{CT}$ \\
\hline 1 & $\mathrm{~T} 1$ & $\mathrm{~T} 2$ & $\mathrm{~T} 1$ & $\mathrm{~T} 1$ & 0.6 & 0.3 & 0.2 \\
\hline 2 & $\mathrm{~T} 1$ & $\mathrm{~T} 2$ & $\mathrm{~T} 1$ & $\mathrm{~T} 1$ & 0.8 & 0.2 & 0.2 \\
\hline 3 & $\mathrm{~T} 1$ & $\mathrm{~T} 2$ & $\mathrm{~T} 2$ & $\mathrm{~T} 1$ & 0.4 & 0.5 & 0.1 \\
\hline 4 & $\mathrm{~T} 1$ & $\mathrm{~T} 3$ & $\mathrm{~T} 1$ & $\mathrm{~T} 1$ & 1.1 & 0.3 & 0.1 \\
\hline 5 & $\mathrm{~T} 2$ & $\mathrm{~T} 3$ & $\mathrm{~T} 3$ & $\mathrm{~T} 3$ & 0.3 & 0.5 & 0.3 \\
\hline 6 & $\mathrm{~T} 1$ & $\mathrm{~T} 1$ & $\mathrm{~T} 3$ & $\mathrm{~T} 1$ & 0.2 & 1.2 & 0.1 \\
\hline 7 & $\mathrm{~T} 1$ & $\mathrm{~T} 1$ & $\mathrm{~T} 2$ & $\mathrm{~T} 1$ & 0.5 & 0.8 & 0.4 \\
\hline 8 & $\mathrm{~T} 3$ & $\mathrm{~T} 1$ & $\mathrm{~T} 2$ & $\mathrm{~T} 3$ & 0.7 & 0.4 & 0.2 \\
\hline 9 & $\mathrm{~T} 2$ & $\mathrm{~T} 2$ & $\mathrm{~T} 1$ & $\mathrm{~T} 2$ & 0.6 & 0.2 & 0.5 \\
\hline 10 & $\mathrm{~T} 1$ & $\mathrm{~T} 1$ & $\mathrm{~T} 2$ & $\mathrm{~T} 1$ & 0.1 & 0.5 & 0.6 \\
\hline 11 & $\mathrm{~T} 2$ & $\mathrm{~T} 1$ & $\mathrm{~T} 2$ & $\mathrm{~T} 2$ & 0.7 & 0.3 & 0.2 \\
\hline 12 & $\mathrm{~T} 1$ & $\mathrm{~T} 1$ & $\mathrm{~T} 1$ & $\mathrm{~T} 2$ & 0.3 & 0.2 & 0.5 \\
\hline
\end{tabular}

MRI, magnetic resonance imaging; PET/CT, positron emission tomography/computed tomography; T, tumor stage.
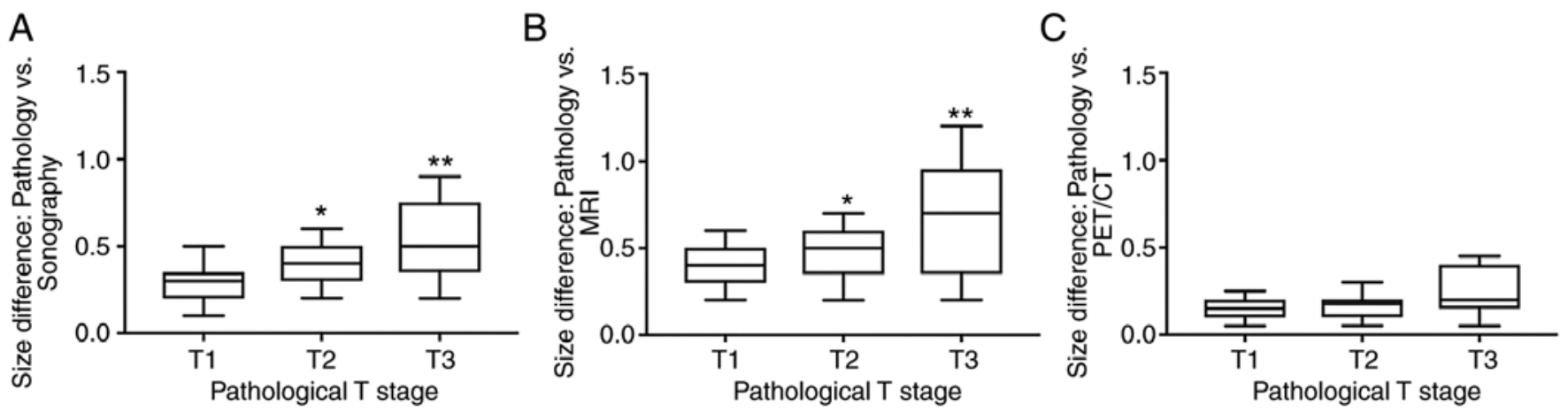

Figure 5. Analysis of tumor size in subgroups of patients with breast cancer classified by tumor stage, according to the 6th Edition of the American Joint Committee on Cancer Staging Manual (22). (A) Sonography, (B) MRI and (C) ${ }^{18} \mathrm{~F}-\mathrm{FDG}$ PET/CT. Significant differences among 3 groups were tested by one-way ANOVA followed by the post-hoc Tukey's test. ${ }^{*} \mathrm{P}<0.05,{ }^{* *} \mathrm{P}<0.01$, compared with ${ }^{18} \mathrm{~F}$-FDG PET/CT group. MRI, magnetic resonance imaging; ${ }^{18} \mathrm{~F}$-FDG PET/CT, positron emission tomography/computed tomography using 2-deoxy-2-fluoro-18-fluoro-D-glucose; T, tumor stage.

solve the issue of tumor size underestimation with the use of sonography to a certain degree. Regarding MRI, a previously published study revealed that pre-operative MRI was associated with lower re-operation rates for close/positive margins $(\mathrm{P}<0.05)(3)$, which affirmed the capacity of MRI for breast tumor assessment. However, a previous study reported an overestimation of tumor size using MRI (33), which is consistent with results obtained in the present study. To the best of our knowledge, the present study is so far the first to compare the reliability and association among ${ }^{18} \mathrm{~F}-\mathrm{FDG}$ PET/CT, MRI and sonography in breast cancer tumor size assessment. The present study, provides further guidance for choosing a more effective diagnostic method for estimating the tumor size in patients with breast cancer.

Since ${ }^{18} \mathrm{~F}-\mathrm{FDG}$ PET/CT was first recommended as a method to estimate tumor size, there are several studies describing the advantages of ${ }^{18} \mathrm{~F}-\mathrm{FDG} \mathrm{PET} / \mathrm{CT}$ for staging breast cancer (34-37). However, only $\mathrm{N}$ staging (lymph node detection) and $\mathrm{M}$ staging (distant metastasis) were took into consideration in these studies, whereas the present study added $\mathrm{T}$ stage for tumor size measurement as well. Furthermore, several studies reported ${ }^{18} \mathrm{~F}$-FDG PET/CT to have low concordance with pathology in terms of $\mathrm{T}$ stage (38-40), while the present study detected a good consistency with pathology and even a lower bias of tumor size. An unclear tumor margin on ${ }^{18} \mathrm{~F}-\mathrm{FDG}$ PET/CT, arising from its inherently low resolution and from the confounding factor of surrounding physiological breast uptake, is likely the main reason why PET/CT is not used for tumor size evaluation (20). Recently, a method using PHL has been reported as reliable for measuring tumor volume in cases of thyroid cancer (41). A recent report suggested that PHL can enhance tumor margin detection on ${ }^{18}$ F-FDG PET/CT (41).

In the present study, the longest tumor diameters obtained with ${ }^{18}$ F-FDG PET/CT scan compared with MRI and sonography, exhibited statistically significant smaller differences and more linear associations with pathological tumor size. This demonstrated that tumor size estimation by ${ }^{18} \mathrm{~F}-\mathrm{FDG}$ 
PET/CT scan can be more accurate than by sonography and MRI and can provide a reliable reference for the decision of an appropriate surgical strategy and prognostic prediction. In T stage assessment, MRI and sonography displayed higher sizes differences (vs. pathology) compared with ${ }^{18} \mathrm{~F}-\mathrm{FDG}$ PET/CT in T2 and T3 stage tumors, but not in T1 stage tumors. Furthermore, MRI assessments resulted in 2 patients being incorrectly upstaged as T3, while this error was avoided in ${ }^{18}$ F-FDG PET/CT assessments. Size differences by ${ }^{18}$ F-FDG PET/CT were also smaller compared to MRI and sonography in 12 incorrectly staged patients. Overestimation of tumor size can be a serious mistake, as it may deprive patients of the opportunity of undergoing breast-conservation therapy, which is a simpler and more superficial type of therapy compared with mastectomy (4). Thus, tumor size estimates using ${ }^{18} \mathrm{~F}$-FDG PET/CT may be more reliable in guiding surgical strategy for large-sized tumors and ${ }^{18} \mathrm{~F}$-FDG PET/CT could be recommended as a better imaging modality in breast cancer tumor estimation compared with other imaging methods.

The present study has several limitations. First, as all the patients are enrolled from a single institution, this may lead to a lack of appropriate representation and selection bias. However, this may not affect the results severely, since the purpose of the present study was simply to evaluate and compare the reliability of a tumor size measurement method using PHL and the included cases exhibited various tumor sizes. Second, investigator influence during malignancy assessment of the results due to previous knowledge of the results derived from other imaging techniques cannot be excluded. Third, the pathological size that was used as a reference standard was solely based on previous pathological reports, while there may be changes in pathological measurement during preservation and preparation of each tissue specimen. Additionally, the bias during sonography should be taken into consideration as it is more likely to be operator-dependent. Last, because of the flat character of imaging analysis and the non-planar growth form of the tumors, only the larger diameter could be measured with the image. Despite the aforementioned limitations, the results of the present study suggest that the ${ }^{18} \mathrm{~F}-\mathrm{FDG}$ PET/CT-based PHL method was superior to breast ultrasound and MRI.

In conclusion, the present study has demonstrated that ${ }^{18}$ F-FDG PET/CT-based PHL can accurately estimate tumor size in patients with breast cancer. Despite the fact that this method may overestimate small size tumors, it is superior to sonography and MRI, with greater association and reliability for pathological tumor size assessment in breast cancer.

\section{Acknowledgements}

Not applicable.

\section{Funding}

No funding was received.

\section{Availability of data and materials}

The datasets used and/or analyzed during the current study are available from the corresponding author on reasonable request.

\section{Authors' contributions}

YW performed patient enrollment according to the exclusion and inclusion criteria of the study, performed experiments and analyzed the data. YL and CX performed experiments, analyzed the data and drafted the manuscript. BL designed and conceived this study. All authors have read and approved the manuscript.

\section{Ethics approval and consent to participate}

The retrospective study was approved by The Ethics Committee of Changxing People's Hospital (Huzhou, China; approval no. CPH 201505). All patients provided signed informed consent.

\section{Patient consent for publication}

All patients enrolled in this study have provided consent for the publication of their examination information.

\section{Competing interests}

The authors declare that they have no competing interests.

\section{References}

1. Jemal A, Bray F, Center MM, Ferlay J, Ward E and Forman D: Global cancer statistics. CA Cancer J Clin 61: 69-90, 2011.

2. Ghoncheh M, Pournamdar Z and Salehiniya H: Incidence and mortality and epidemiology of breast cancer in the world. Asian Pac J Cancer Prev 17 (S3): 43-46, 2016.

3. McGhan LJ, Wasif N, Gray RJ, Giurescu ME, Pizzitola VJ, Lorans R, Ocal IT, Stucky CC and Pockaj BA: Use of preoperative magnetic resonance imaging for invasive lobular cancer: Good, better, but maybe not the best? Ann Surg Oncol 17 (Suppl 3): 255-262, 2010.

4. Pop CF, Stanciu-Pop C, Drisis S, Radermeker M, Vandemerckt C, Noterman D, Moreau M, Larsimont D, Nogaret JM and Veys I: The impact of breast MRI workup on tumor size assessment and surgical planning in patients with early breast cancer. Breast J 24: 927-933, 2018.

5. Miller AB, Baines CJ, To T and Wall C: Canadian National Breast Screening Study: 1. Breast cancer detection and death rates among women aged 40 to 49 years. CMAJ 147: 1459-1476, 1992.

6. Don S, Choi E and Min D: Breast mass segmentation in digital mammography using graph cuts. In: Convergence and Hybrid Information Technology. Lee G, Howard D and Ślęzak D (eds). Vol. 206. Springer Berlin, Heidelberg, pp88-96, 2011.

7. Brem RF, Ioffe M, Rapelyea JA, Yost KG, Weigert JM, Bertrand ML and Stern LH: Invasive lobular carcinoma: Detection with mammography, sonography, MRI, and breast-specific gamma imaging. AJR Am J Roentgenol 192: 379-383, 2009

8. Sarno A, Mettivier G and Russo P: Dedicated breast computed tomography: Basic aspects. Med Phys 42: 2786-2804, 2015.

9. Onesti JK, Mangus BE, Helmer SD and Osland JS: Breast cancer tumor size: Correlation between magnetic resonance imaging and pathology measurements. Am J Surg 196: 844-848, discussion 849-850, 2008 .

10. Narod SA: Age of diagnosis, tumor size, and survival after breast cancer: Implications for mammographic screening. Breast Cancer Res Treat 128: 259-266, 2011.

11. Nyström L, Rutqvist LE, Wall S, Lindgren A, Lindqvist M, Rydén S, Andersson I, Bjurstam N, Fagerberg G, Frisell J, et al: Breast cancer screening with mammography: Overview of Swedish randomised trials. Lancet 341: 973-978, 1993.

12. Pilewskie M, Hirsch A, Eaton A, Stempel M and Gemignani ML: Breast cancer in the elderly: Is MRI helpful? Breast J 21: 651-657, 2015. 
13. Hatada I, Hayashizaki Y, Hirotsune S, Komatsubara H and Mukai T: A genomic scanning method for higher organisms using restriction sites as landmarks. Proc Natl Acad Sci USA 88: 9523-9527, 1991.

14. Boetes C, Veltman J, van Die L, Bult P, Wobbes $T$ and Barentsz JO: The role of MRI in invasive lobular carcinoma. Breast Cancer Res Treat 86: 31-37, 2004.

15. Lo GG, Ai V, Chan JKF, Li KW, Cheung PSY, Wong TT, Ma M, Lee $\mathrm{R}$ and Chien D: Diffusion-weighted magnetic resonance imaging of breast lesions: First experiences at $3 \mathrm{~T}$. J Comput Assist Tomogr 33: 63-69, 2009.

16. Kuhl CK, Schrading S, Leutner CC, Morakkabati-Spitz N, Wardelmann E, Fimmers R, Kuhn $\mathrm{W}$ and Schild HH: Mammography, breast ultrasound, and magnetic resonance imaging for surveillance of women at high familial risk for breast cancer. J Clin Oncol 23: 8469-8476, 2005.

17. Burkett BJ and Hanemann CW: A review of supplemental screening ultrasound for breast cancer: Certain populations of women with dense breast tissue may benefit. Acad Radiol 23: 1604-1609, 2016.

18. Paydary K, Seraj SM, Zadeh MZ, Emamzadehfard S, Shamchi SP, Gholami S, Werner TJ and Alavi A: The evolving role of FDG-PET/CT in the diagnosis, staging, and treatment of breast cancer. Mol Imaging Biol 21: 1-10, 2019.

19. Uematsu T, Kasami M and Yuen S: Comparison of FDG PET and MRI for evaluating the tumor extent of breast cancer and the impact of FDG PET on the systemic staging and prognosis of patients who are candidates for breast-conserving therapy. Breast Cancer 16: 97-104, 2009.

20. Nogami Y, Iida M, Banno K, Kisu I, Adachi M, Nakamura K, Umene K, Masuda K, Tominaga E, Tanaka K, et al: Application of FDG-PET in cervical cancer and endometrial cancer: Utility and future prospects. Anticancer Res 34: 585-592, 2014.

21. Park SH, Seo M, Choi HJ, Bae K, Bang M and Jun S: More accurate than MRI measurement of tumor size in breast cancer by using the peri-tumoral halo uptake layer method of the 18F-FDG PET/CT scan. Hell J Nucl Med 21: 108-114, 2018.

22. Jun S, Kim H and Nam HY: A new method for segmentation of FDG PET metabolic tumour volume using the peritumoural halo layer and a 10-step colour scale. A study in patients with papillary thyroid carcinoma. Nucl Med (Stuttg) 54: 272-285, 2015.

23. Singletary SE, Allred C, Ashley P, Bassett LW, Berry D, Bland KI, Borgen PI, Clark GM, Edge SB, Hayes DF, et al: Staging system for breast cancer: revisions for the 6th edition of the AJCC Cancer Staging Manual. Surg Clin North Am 83: 803-819, 2003.

24. Mendelson E, Baum J, Berg W, Merritt C and Rubin E: Breast imaging reporting and data system. BI-RADS, 2003.

25. Zhengfeng L: Diagnostic ultrasound system quality control testing methods. Chin Med Dev 07: 27-32, 2011 (In Chinese).

26. Hengdi W: Image quality control standards and procedures of MRI devices. POSTRUM 28: 19-23, 2013 (In Chinese)

27. Jun Y, Jianwei W, Shuyue A and Wei H: Quality control and administration of PET/CT. Chin Med Euipment J 27: 71-73, 2006 (In Chinese).

28. Grimsby GM, Gray R, Dueck A, Carpenter S, Stucky CC, Aspey H, Giurescu ME and Pockaj B: Is there concordance of invasive breast cancer pathologic tumor size with magnetic resonance imaging? Am J Surg 198: 500-504, 2009.

29. Luparia A, Mariscotti G, Durando M, Ciatto S, Bosco D, Campanino PP, Castellano I, Sapino A and Gandini G: Accuracy of tumour size assessment in the preoperative staging of breast cancer: Comparison of digital mammography, tomosynthesis, ultrasound and MRI. Radiol Med (Torino) 118: 1119-1136, 2013.
30. Lai HW, Chen DR, Wu YC, Chen CJ, Lee CW, Kuo SJ, Chen ST and Wu HK: Comparison of the diagnostic accuracy of magnetic resonance imaging with sonography in the prediction of breast cancer tumor size: A concordance analysis with histopathologically determined tumor size. Ann Surg Oncol 22: 3816-3823, 2015.

31. Gruber I, Rueckert M, Kagan K, Staebler A, Siegmann KC, Hartkopf A, Wallwiener D and Hahn M: Measurement of tumour size with mammography, sonography and magnetic resonance imaging as compared to histological tumour size in primary breast cancer. BMC Cancer 13: 328, 2013.

32. Hieken TJ, Harrison J, Herreros J and Velasco JM: Correlating sonography, mammography, and pathology in the assessment of breast cancer size. Am J Surg 182: 351-354, 2001.

33. Jethava A, Ali S, Wakefield D, Crowell R, Sporn J and Vrendenburgh J: Diagnostic accuracy of MRI in predicting breast tumor size: Comparative analysis of MRI vs histopathological assessed breast tumor size. Conn Med 79: 261-267, 2015.

34. Caresia Aroztegui AP, García Vicente AM, Alvarez Ruiz S, Delgado Bolton RC, Orcajo Rincon J, Garcia Garzon JR, de Arcocha Torres M and Garcia-Velloso MJ: 18F-FDG PET/CT in breast cancer: Evidence-based recommendations in initial staging. Tumour Biol 39: 1010428317728285, 2017.

35. Pritchard KI, Julian JA, Holloway CM, McCready D, Gulenchyn KY, George R, Hodgson N, Lovrics P, Perera F, Elavathil L, et al: Prospective study of $2-\left[{ }^{18} \mathrm{~F}\right]$ fluorodeoxyglucose positron emission tomography in the assessment of regional nodal spread of disease in patients with breast cancer: An Ontario clinical oncology group study. J Clin Oncol 30: 1274-1279, 2012.

36. Riegger C, Herrmann J, Nagarajah J, Hecktor J, Kuemmel S, Otterbach F, Hahn S, Bockisch A, Lauenstein T, Antoch G, et al: Whole-body FDG PET/CT is more accurate than conventional imaging for staging primary breast cancer patients. Eur J Nucl Med Mol Imaging 39: 852-863, 2012.

37. Avril N, Rosé CA, Schelling M, Dose J, Kuhn W, Bense S, Weber W, Ziegler S, Graeff H and Schwaiger M: Breast imaging with positron emission tomography and fluorine-18 fluorodeoxyglucose: Use and limitations. J Clin Oncol 18: 3495-3502, 2000.

38. Hyun SH, Ahn HK, Park YH, Im YH, Kil WH, Lee JE, Nam SJ, Cho EY and Choi JY: Volume-based metabolic tumor response to neoadjuvant chemotherapy is associated with an increased risk of recurrence in breast cancer. Radiology 275: 235-244, 2015.

39. Kim J, Yoo SW, Kang SR, Cho SG, Oh JR, Chong A, Min JJ, Bom HS, Yoon JH and Song HC: Prognostic significance of metabolic tumor volume measured by (18)F-FDG PET/CT in operable primary breast cancer. Nucl Med Mol Imaging 46: 278-285, 2012.

40. Oh JR, Seo JH, Chong A, Min JJ, Song HC, Kim YC and Bom HS: Whole-body metabolic tumour volume of $18 \mathrm{~F}-\mathrm{FDG}$ PET/CT improves the prediction of prognosis in small cell lung cancer. Eur J Nucl Med Mol Imaging 39: 925-935, 2012.

41. Jun S, Park JG and Seo Y: Accurate FDG PET tumor segmentation using the peritumoral halo layer method: A study in patients with esophageal squamous cell carcinoma. Cancer Imaging 18: 35, 2018.

(i) (5) $\odot$ This work is licensed under a Creative Commons Attribution-NonCommercial-NoDerivatives $\quad 4.0$ International (CC BY-NC-ND 4.0) License. 